\title{
LU Decomposition Method for Solving Fully Fuzzy Linear System with Trapezoidal Fuzzy Numbers
}

\author{
S. Radhakrishnan, R. Sattanathan and P. Gajivaradhan
}

\begin{abstract}
System of simultaneous linear equations plays a vital role in mathematics, Operations Research, Statistics, Physics, Engineering and Social Sciences etc. In many applications at least some of the system's parameters and measurements are represented by fuzzy numbers rather than crisp numbers. Therefore it is imperative to develop mathematical models and numerical procedures to solve such a fuzzy linear system. The general model of a fuzzy linear system whose coefficient matrix is crisp and the right hand side column is an arbitrary fuzzy vector. In the fully fuzzy linear system all the parameters are considered to be fuzzy numbers. Since triangular fuzzy numbers is a special case of trapezoidal fuzzy numbers, hence in this paper we considered fully fuzzy linear system with trapezoidal fuzzy numbers. LU decomposition method for a crisp matrix is well known in solving linear system of equations. We discuss $L U$ decomposition of the coefficient matrix of the fully fuzzy linear system, in which the coefficients are trapezoidal fuzzy numbers.
\end{abstract}

Keywords--- Fully Fuzzy Linear System, LU Decomposition, Trapezoidal Numbers

\section{INTRODUCTION}

$\mathrm{T}$ HE concept of fuzzy numbers and fuzzy arithmetic operations were first introduced by Zadeh. M. Fridamn, M. Ming , M. Kandel [1] introduced a general model for solving a fuzzy $\mathrm{n} \times \mathrm{n}$ linear system whose coefficient matrix is crisp and the right hand side column is a fuzzy vector of positive fuzzy numbers. M. Dehghan, B. Hashemi, M. Ghatee, [3] are solved $\mathrm{n} \times \mathrm{n}$ fully fuzzy linear system using direct method, Cramer's rule, Gauss Elimination, Doolittle \& Crout factorization methods and Linear programming approach. Amit Kumar, Neetu, Abhinav Bansal [8] discussed consistency of the fully fuzzy linear system and the nature of solutions. S.H. Neseri, M. Sohrabi, E. Ardil [5] Proposed a method for solving fully fuzzy linear systems by certain decomposition (LU) of the coefficient matrix with triangular

S. Radhakrishnan, Research Scholar, University of madras, Assistant Professor, Department of Mathematics, D.G.Vaishnav College, Chennai, India. E-mail:adithyakrish18@gmail.com

R. Sattanathan, Associate Professor \& Head, Department of Mathematics, D.G.Vaishnav College, Chennai, India.

P. Gajivaradhan, Associate Professor, Department of Mathematics, Pachaiyapp's College, Chennai, India. fuzzy numbers. M. Mosleh, M. Otadi and A. Khanmirzaie [6] introduced ST decomposition for $2 \times 2$ fully fuzzy linear systems with triangular fuzzy numbers. P. SenthilKumar, G Rajendran, [9] have solved $\mathrm{n} \times \mathrm{n}$ fully fuzzy linear system using Cholesky method. Amit kumar, Abhinav Bansal, Neetu [10] are bring in a new method for finding the non negative solution of fully fuzzy linear system without any restriction on the coefficient matrix. kumar, Neetu, Abhinav Bansal [11] are put in a new method for finding the non negative solution of the $\mathrm{m} \times \mathrm{n}$ fully fuzzy linear system without any restriction on the coefficient matrix using Linear programming problem method. Nasseri et al. [7] proposed Greville's method to find the positive solution of fully fuzzy linear system. T. Allahyiranloo [2] proposed solution of a fuzzy linear system by iterative methods such as Jacobi and Gauss Seidel methods. A.K. Shyamal and M. Pal [4] discussed Triangular Fuzzy matrices.

The structure of this paper is organized as follows

In Section II, we present some basic concepts of fuzzy set theory and define a fully fuzzy linear system of equations. In Section III, A numerical method for computing the solution of Fully fuzzy linear system is discussed. Section IV deals with a Numerical example to illustrate the above method. Section V ends this paper with conclusion and References.

\section{PRELIMINARIES}

- Definition 2.1: A fuzzy subset $\widetilde{\mathrm{A}}$ of $\mathrm{R}$ is defined by its membership function $\mu_{\widetilde{A}}: R \rightarrow[0,1]$, which assigns a real number $\mu_{\widetilde{A}}$ in the interval $[0,1]$, to each element $x \in R$, Where the value of $\mu_{\widetilde{A}}$ at $x$ shows the grade of membership of $x$ in $\widetilde{A}$.

- Definition 2.2: A fuzzy number $\widetilde{\mathrm{A}}=(\mathrm{m}, \mathrm{n}, \alpha, \beta)$ is said to be a trapezoidal fuzzy number if its membership function is given by

$$
\mu_{\tilde{A}}(x)=\left\{\begin{array}{cc}
1-\frac{m-x}{\alpha}, & m-\alpha \leq x \leq m, \alpha>0 \\
1, & m<x<n \\
1-\frac{x-n}{\beta}, & n \leq x \leq n+\beta, \beta>0 \\
0, & \text { otherwise }
\end{array}\right.
$$

- Definition 2.3: A fuzzy number $\tilde{A}$ is called positive (negative), denoted by $\widetilde{\mathrm{A}}>0(\widetilde{\mathrm{A}}<0)$, if its membership function $\mu_{\widetilde{\mathrm{A}}}(\mathrm{x})$ satisfies $\mu_{\widetilde{\mathrm{A}}}(\mathrm{x})=0, \forall \mathrm{x} \leq$ $0(\forall \mathrm{x} \geq 0)$. 
- Definition 2.4: Two fuzzy numbers $\widetilde{\mathrm{A}}=(\mathrm{m}, \mathrm{n}, \alpha, \beta)$ and $\widetilde{B}=(p, q, \gamma, \delta)$ are said to be equal if and only if $\mathrm{m}=\mathrm{p}, \mathrm{n}=\mathrm{q}, \alpha=\gamma$, and $\beta=\delta$.

- Definition 2.5: A Trapezoidal fuzzy number $\widetilde{\mathrm{A}}=(\mathrm{m}$, $\mathrm{n}, \alpha, \beta)$ is said to be zero trapezoidal fuzzy number if and only if $m=0, n=0, \alpha=0, \beta=0$.

- Definition 2.6: Let $\widetilde{\mathrm{A}}=\left(\tilde{\mathrm{a}}_{\mathrm{ij}}\right)$ and $\widetilde{\mathrm{B}}=\left(\tilde{\mathrm{b}}_{\mathrm{ij}}\right)$ be two $\mathrm{mX} \mathrm{n}$ and $\mathrm{nX} \mathrm{p}$ fuzzy matrices. We define $\widetilde{A} \otimes \tilde{B}=\tilde{C}=\left(\tilde{c}_{i j}\right)$ Which is the $\mathrm{mX} \mathrm{p}$ matrix where

$$
\tilde{C}_{i j}=\sum_{k=1,2 \ldots . n}^{\oplus} \tilde{a}_{i k} \otimes \tilde{b}_{k j}
$$

- Definition 2.7: Arithmetic operations on trapezoidal numbers

Let $\widetilde{\mathrm{A}}_{1}=(\mathrm{m}, \mathrm{n}, \alpha, \beta)$ and $\widetilde{\mathrm{A}}_{2}=(\mathrm{p}, \mathrm{q}, \gamma, \delta)$ be two trapezoidal fuzzy numbers then

$$
\begin{aligned}
& \text { (i) } \begin{aligned}
\widetilde{\mathrm{A}}_{1} \oplus \widetilde{\mathrm{A}}_{2}=(\mathrm{m}, \mathrm{n}, \alpha, \beta) \oplus(\mathrm{p}, \mathrm{q}, \gamma, \delta) \\
=(\mathrm{m}+\mathrm{p}, \mathrm{n}+\mathrm{q}, \alpha+\gamma, \beta+\delta)
\end{aligned} \\
& \text { (ii) } \begin{array}{r}
-\widetilde{\mathrm{A}}_{1}=-(\mathrm{m}, \mathrm{n}, \alpha, \beta)=(-\mathrm{n},-\mathrm{m}, \beta, \alpha) \\
\text { (iii) } \widetilde{\mathrm{A}}_{1} \geq 0 \text { and } \widetilde{\mathrm{A}}_{1} \geq \mathrm{o} \text { then } \\
\widetilde{\mathrm{A}}_{1} \otimes \widetilde{\mathrm{A}}_{2}=(\mathrm{m}, \mathrm{n}, \alpha, \beta) \otimes(\mathrm{p}, \mathrm{q}, \gamma, \delta) \\
\cong(\mathrm{mp}, \mathrm{nq}, \mathrm{m} \gamma+\mathrm{p} \alpha, \mathrm{n} \delta+\mathrm{q} \beta)
\end{array}
\end{aligned}
$$

- Definition 2.8: $A$ matrix $\widetilde{\mathrm{A}}=\left(\widetilde{\mathrm{a}}_{\mathrm{ij}}\right)$ is called a fuzzy matrix, if each element of $\widetilde{\mathrm{A}}$ is a fuzzy number. A fuzzy matrix $\tilde{A}$ will be positive and denoted by $\tilde{A}>0$, if each element of $\tilde{A}$ be positive. We may represent n X n fuzzy matrix $\tilde{A}=\left(\tilde{a}_{i j}\right)_{\mathrm{nXn}}$ such that $\tilde{a}_{i j}=\left(a_{i j}, b_{i j}, m_{i j}, n_{i j}\right)$, with the new notation $\tilde{A}=(A, B, M, N)$, where $\mathrm{A}=\left(a_{i j}\right), \quad B=\left(b_{i j}\right)$ $M=\left(m_{i j}\right), N=\left(n_{i j}\right)$ are four n $\mathrm{X}$ n crisp matrices.

- Definition 2.9: A square fuzzy matrix $\widetilde{\mathrm{A}}=\left(\widetilde{\mathrm{a}}_{\mathrm{ij}}\right)$ will be an upper triangular fuzzy matrix, if $\tilde{\mathrm{a}}_{\mathrm{ij}}=\tilde{0}=$ $(0,0,0,0) \forall \mathrm{i}>\mathrm{j}$, and a square fuzzy matrix $\widetilde{\mathrm{A}}=\left(\widetilde{\mathrm{a}}_{\mathrm{ij}}\right)$ will be a lower triangular fuzzy matrix, if $\tilde{\mathrm{a}}_{\mathrm{ij}}=\tilde{0}=(0,0,0,0) \forall \mathrm{i}<\mathrm{j}$.

- Definition 2.10: Consider the $\mathrm{n} X \mathrm{n}$ fuzzy linear system of equations

$\left(\tilde{a}_{11} \otimes \tilde{x}_{1}\right) \oplus\left(\tilde{a}_{12} \otimes \tilde{x}_{2}\right) \oplus \ldots \ldots \ldots \oplus\left(\tilde{a}_{1 n} \otimes \tilde{x}_{n}\right)=\tilde{b}_{1}$

$\left(\tilde{a}_{21} \otimes \tilde{x}_{1}\right) \oplus\left(\tilde{a}_{22} \otimes \tilde{x}_{2}\right) \oplus \ldots \ldots \ldots \oplus\left(\tilde{a}_{2 n} \otimes \tilde{x}_{n}\right)=\tilde{b}_{2}$

$\left(\tilde{a}_{n 1} \otimes \tilde{x}_{1}\right) \oplus\left(\tilde{a}_{n 2} \otimes \tilde{x}_{2}\right) \oplus \ldots \ldots \ldots \oplus\left(\tilde{a}_{n n} \otimes \tilde{x}_{n}\right)=\tilde{b}_{n}$

The matrix form of the above equations is $\tilde{A} \otimes \tilde{x}=\tilde{b}$

Where the coefficient matrix $\widetilde{A}=\left(\tilde{a}_{i j}\right), 1 \leq \mathrm{i}, \mathrm{j} \leq \mathrm{n}$ is a $\mathrm{X} \mathrm{n}$ fuzzy matrix and $\tilde{x}_{j} \tilde{b}_{j} \in F(R)$. This system is called a fully fuzzy linear system.

In this paper $\tilde{A} \otimes \tilde{x}=\tilde{b}$ where $\tilde{A}=(A, B, M, N), \tilde{x}=$ $(x, y, z, w) \geq 0$ and $\tilde{b}=(b, g, h, k) \geq 0$

We have

$$
(\mathrm{A}, \mathrm{B}, \mathrm{M}, \mathrm{N}) \otimes(\mathrm{x}, \mathrm{y}, \mathrm{z}, \mathrm{w})=(\mathrm{b}, \mathrm{g}, \mathrm{h}, \mathrm{k})
$$

Using 2.7 (iii) we have

$$
(\mathrm{Ax}, \mathrm{By}, \mathrm{Az}+\mathrm{Mx}, \mathrm{Bw}+\mathrm{Ny})=(\mathrm{b}, \mathrm{g}, \mathrm{h}, \mathrm{k})
$$

Using 2.4 we have

$\mathrm{Ax}=\mathrm{b}$

$\mathrm{By}=\mathrm{g}$

$\mathrm{Az}+\mathrm{Mx}=\mathrm{h}$

$\mathrm{Bw}+\mathrm{Ny}=\mathrm{k}$

By assuming that $\mathrm{A}$ and $\mathrm{B}$ are non-singular matrices we have

$$
\begin{aligned}
& x=A^{-1} b \\
& y=B^{-1} g \\
& z=A^{-1}(h-M x) \\
& w=B^{-1}(k-N y)
\end{aligned}
$$

\section{A LU DECOMPOSITION METHOD FOR SOLVING FULLY FUZZY LINEAR SYSTEMS FOR TRAPEZOIDAL FUZZY NUMBERS}

\section{A. Theorem 3.1}

Let $\mathrm{A}$ be an $\mathrm{n} \mathrm{X} \mathrm{n}$ matrix with all non-zero leading principal minors. Then $\mathrm{A}$ has a unique factorization $\mathrm{A}=\mathrm{L} \mathrm{U}$, Where $\mathrm{L}$ is unit lower triangular and $\mathrm{U}$ is upper triangular.

Assume that $\tilde{A}=(A, B, M, N)$, where A and B are the full rank crisp matrices. Then if we let

$$
\left(L_{1}, L_{2}, 0,0\right) \otimes\left(U_{1}, U_{2}, U_{3}, U_{4}\right)=(A, B, M, N)
$$

Using 2.7 (iii) we have

$$
\begin{aligned}
& \left(L_{1} U_{1}, L_{2} U_{2}, L_{1} U_{3}, L_{2} U_{4}\right)=(A, B, M, N) \\
& L_{1} U_{1}=A \Rightarrow U_{1}=L_{1}^{-1} A \\
& L_{2} U_{2}=B \Rightarrow U_{2}=L_{2}^{-1} B \\
& L_{1} U_{3}=M \Rightarrow U_{3}=L_{1}^{-1} M \\
& L_{2} U_{4}=N \Rightarrow U_{4}=L_{2}^{-1} N
\end{aligned}
$$

Where matrices $L_{1}$ and $L_{2}$ are lower triangular crisp matrices and matrices $U_{1}$ and $U_{2}$ are upper triangular crisp matrices.

Consider the fully fuzzy linear systems.

We are going to construct a method for solving fully fuzzy linear systems $\tilde{A} \otimes \tilde{x}=\tilde{b}$, Where $\tilde{A}=(A, B, M, N)$, $\tilde{x}=(x, y, z, w), \tilde{b}=(b, g, h, k)$, that is

$$
\begin{aligned}
& (A, B, M, N) \otimes(x, y, z, w)=(b, g, h, k) \\
& \left(L_{1} U_{1}, L_{2} U_{2}, L_{1} U_{3}, L_{2} U_{4}\right) \otimes(x, y, z, w)=(b, g, h, k)
\end{aligned}
$$

Using 2.7 (iii) we have 


$$
\begin{aligned}
& \left(L_{1} U_{1} x, L_{2} U_{2} y, L_{1} U_{1} z+L_{1} U_{3} x, L_{2} U_{2} w+L_{2} U_{4} y\right)= \\
& (b, g, h, k)
\end{aligned}
$$

The current system by use of Definition 2.4 can be rewrite as follows

$$
\begin{aligned}
& L_{1} U_{1} x=b \\
& L_{2} U_{2} y=g \\
& L_{1} U_{1} z+L_{1} U_{3} x=h \\
& L_{2} U_{2} w+L_{2} U_{4} y=k
\end{aligned}
$$

\section{Therefore}

$x=U_{1}^{-1} L_{1}^{-1} b$

$y=U_{2}^{-1} L_{2}^{-1} g$

$z=U_{1}^{-1} L_{1}^{-1}\left(h-L_{1} U_{3} x\right)$

$w=U_{2}^{-1} L_{2}^{-1}\left(k-L_{2} U_{4} y\right)$

\section{B. Algorithm}

\section{(Fully Fuzzy Linear Systems Solver)}

Step 1: Assume that $\tilde{A}=(A, B, M, N)$, Where A and B are the full rank crisp matrices.

Compute LU- decomposition for crisp matrix $A=L_{1} U_{1}$ and $B=L_{2} U_{2}$

Step 2: Set $L_{3}=0$ and $L_{4}=0$

Step 3: Compute $U_{3}=L_{1}^{-1} M$

Step 4: Compute $U_{4}=L_{2}^{-1} N$

Step 5: Compute the solution of the fully fuzzy system $(\mathrm{A}, \mathrm{B}, \mathrm{M}, \mathrm{N}) \otimes(\mathrm{x}, \mathrm{y}, \mathrm{z}, \mathrm{w})=(\mathrm{b}, \mathrm{g}, \mathrm{h}, \mathrm{k})$ as follows

$x=U_{1}^{-1} L_{1}^{-1} b$

$y=U_{2}^{-1} L_{2}^{-1} g$

$z=U_{1}^{-1} L_{1}^{-1}\left(h-L_{1} U_{3} x\right)$

$w=U_{2}^{-1} L_{2}^{-1}\left(k-L_{2} U_{4} y\right)$

\section{NUMERICAL EXAMPLE}

Consider the following fully fuzzy linear system

$(3,6,2,2) \otimes\left(x_{1}, y_{1}, z_{1}, w_{1}\right) \oplus(4,6,1,2) \otimes\left(x_{2}, y_{2}, z_{2}, w_{2}\right)=$ $(27,66,26,58)$

$(4,5,1,1) \otimes\left(x_{1}, y_{1}, z_{1}, w_{1}\right) \oplus(5,8,1,2) \otimes\left(x_{2}, y_{2}, z_{2}, w_{2}\right)=$ $(35,70,25,55)$

Solution: The given fully fuzzy linear system can be written as

$$
\left[\begin{array}{ll}
(3,6,2,2) & (4,6,1,2) \\
(4,5,1,1) & (5,8,1,2)
\end{array}\right]\left[\begin{array}{c}
\left(x_{1}, y_{1}, z_{1}, w_{1}\right) \\
\left(x_{2}, y_{2}, z_{2}, w_{2}\right)
\end{array}\right]=\left[\begin{array}{l}
(27,66,26,58) \\
(35,70,25,55)
\end{array}\right]
$$

$$
\begin{aligned}
& x=A^{-1} b=U_{1}^{-1} L_{1}^{-1} b=\left[\begin{array}{l}
5 \\
3
\end{array}\right] \\
& y=B^{-1} g=U_{2}^{-1} L_{2}^{-1} g=\left[\begin{array}{l}
6 \\
5
\end{array}\right] \\
& z=A^{-1}(h-M x)=U_{1}^{-1} L_{1}^{-1}\left(h-L_{1} U_{3} x\right)=\left[\begin{array}{l}
3 \\
1
\end{array}\right] \\
& w=B^{-1}(k-N y)=U_{2}^{-1} L_{2}^{-1}\left(k-L_{2} U_{4} y\right)=\left[\begin{array}{l}
3 \\
3
\end{array}\right] \\
& \tilde{x}_{1}=(5,6,3,3) \text { and } \tilde{x}_{2}=(3,5,1,3)
\end{aligned}
$$

\section{CONCLUSION}

In this paper, the solution of fully fuzzy linear system is obtained by LU decomposition method in the form of trapezoidal fuzzy number matrices. We assumed $\square_{3,} \square_{4}=0$ because, it will reduce the computational work and our aim is to find a lower triangular structure for Crisp matrices for A and $\mathrm{B}$ not for $\mathrm{M}$ and $\mathrm{N}$ [3].

\section{REFERENCES}

[1] M. Fridamn, M. Ming , M. Kandel, Fuzzy linear systems, fuzzy sets and systems 96, 1998 201-209.

[2] T. Allahviranloo, Numerical methods for fuzzy system of linear equations, Applied mathematics and Computation, Vol,155, 2004, 493502.

[3] M. Dehghan, B. Hashemi, M. Ghatee, computational methods for solving fully fuzzy linear systems, Applied Mathematics and Computation, 179, 2006, 328-343.

[4] A.K. Shyamal and M. Pal, Triangular Fuzzy matrices, Iranian Journal of Fuzzy systems.Vol.4, No.1,2007, 75-87.

[5] S.H. Nasseri, M. Sohrabi, E. Ardil, Solving fully fuzzy linear systems by use of a Certain Decomposition of the Coefficient Matrix, World Academy of Science, Engineering and Technology, 43, 2008, 787-789.

[6] M. Mosleh, M.Otadi, A. Khanmirzaie, Decomposition Method for solving fully fuzzy linear systems, Iranian Journal of Optimization 1, 2009, 188-198.

[7] S.H. Nasseri, M. Matinfar, Z. Kheiri, Greville's Method for the fully fuzzy linear system of equations, Advances in fuzzy set and systems, 4 ,2009, 301-311.

[8] Amit kumar, Neetu, Abhinav Bansal, A new method to solve fully fuzzy linear system with trapezoidal fuzzy numbers, Canadian Journal on Science and Engineering Mathematics Vol.1, No.3, August 2010, 45-56.

[9] P. SenthilKumar, G Rajendran, New approach to solve symmetric fully fuzzy linear systems, Indian academy of sciences, Sadhana, Vol.36, Part 6, December 2011, 933-940.

[10] Amit kumar, Abhinav Bansal, Neetu, Solution of fully fuzzy linear system with arbitrary coefficients, International journal of Applied Mathematics and Computation, Vol.3(3), 2011, 232-237.

[11] Amit kumar, Neetu, Abhinav Bansal, A new approach for solving fully fuzzy linear systems.

$$
\begin{aligned}
A & =\left[\begin{array}{ll}
3 & 4 \\
4 & 5
\end{array}\right], B=\left[\begin{array}{ll}
6 & 6 \\
5 & 8
\end{array}\right], M=\left[\begin{array}{ll}
2 & 1 \\
1 & 1
\end{array}\right], N=\left[\begin{array}{ll}
2 & 2 \\
1 & 2
\end{array}\right] \\
b & =\left[\begin{array}{l}
27 \\
35
\end{array}\right], g=\left[\begin{array}{ll}
66 \\
70
\end{array}\right], h=\left[\begin{array}{ll}
26 \\
25
\end{array}\right], k=\left[\begin{array}{ll}
58 \\
55
\end{array}\right] \\
A & =L_{1} U_{1}=\left[\begin{array}{ll}
1 & 0 \\
4 / 3 & 1
\end{array}\right]\left[\begin{array}{ll}
3 & 4 \\
0 & -1 / 3
\end{array}\right] \\
B & =L_{2} U_{2}=\left[\begin{array}{ll}
1 & 0 \\
5 / 6 & 1
\end{array}\right]\left[\begin{array}{ll}
6 & 6 \\
0 & 3
\end{array}\right] \\
U_{3} & =L_{1}^{-1} M=\left[\begin{array}{ll}
1 & 0 \\
-4 / 3 & 1
\end{array}\right]\left[\begin{array}{ll}
2 & 1 \\
1 & 1
\end{array}\right]=\left[\begin{array}{cc}
2 & 1 \\
-5 / 3 & -1 / 3
\end{array}\right] \\
U_{4} & =L_{2}^{-1} N=\left[\begin{array}{ll}
1 & 0 \\
-5 / 6 & 1
\end{array}\right]\left[\begin{array}{ll}
2 & 2 \\
1 & 2
\end{array}\right]=\left[\begin{array}{cc}
2 & 2 \\
-2 / 3 & 1 / 3
\end{array}\right]
\end{aligned}
$$

